PROCEEDINGS OF THE

AMERICAN MATHEMATICAL SOCIETY

Volume 139, Number 6, June 2011, Pages 2175-2182

S 0002-9939(2010)10747-5

Article electronically published on November 30, 2010

\title{
UNIQUENESS OF REFLECTIONLESS JACOBI MATRICES AND THE DENISOV-RAKHMANOV THEOREM
}

\author{
CHRISTIAN REMLING
}

(Communicated by Walter Van Assche)

\begin{abstract}
If a Jacobi matrix $J$ is reflectionless on $(-2,2)$ and has a single $a_{n_{0}}$ equal to 1 , then $J$ is the free Jacobi matrix $a_{n} \equiv 1, b_{n} \equiv 0$. The paper discusses this result and its generalization to arbitrary sets and presents several applications, including the following: if a Jacobi matrix has some portion of its $a_{n}$ 's close to 1 , then one assumption in the Denisov-Rakhmanov Theorem can be dropped.
\end{abstract}

\section{StATEMENT OF RESUlts}

A Jacobi matrix is a difference operator of the following type:

$$
(J u)_{n}=a_{n} u_{n+1}+a_{n-1} u_{n-1}+b_{n} u_{n} .
$$

Here $a_{n}>0$ and $b_{n} \in \mathbb{R}$, and we also always assume that $a, b$ are bounded sequences.

Alternatively, one can represent $J$ by a tridiagonal matrix with respect to the standard basis of $\ell^{2}(\mathbb{Z})$ :

$$
J=\left(\begin{array}{ccccccc}
\ddots & \ddots & \ddots & & & & \\
& a_{-2} & b_{-1} & a_{-1} & & & \\
& & a_{-1} & b_{0} & a_{0} & & \\
& & & a_{0} & b_{1} & a_{1} & \\
& & & & \ddots & \ddots & \ddots
\end{array}\right) .
$$

Half-line operators $J_{+}$, on $\ell^{2}\left(\mathbb{Z}_{+}\right)$, say, are defined similarly, by considering a suitable truncation of this matrix.

The case $a_{n} \equiv 1$ is of particular interest; these operators are called (discrete) Schrödinger operators.

The Denisov-Rakhmanov (DR) Theorem [5, 10] says the following:

Theorem $1.1([5])$. Suppose that $(1) \sigma_{e s s}\left(J_{+}\right)=[-2,2]$ and $(2) \Sigma_{a c}\left(J_{+}\right)=[-2,2]$. Then $a_{n} \rightarrow 1, b_{n} \rightarrow 0$ as $n \rightarrow \infty$.

Here $\Sigma_{a c}\left(J_{+}\right)$denotes an essential support of the absolutely continuous part of the spectral measure $\nu_{+}$of $J_{+}$; in other words, $\nu_{+, a c}(M)=0$ if and only if $\left|M \cap \Sigma_{a c}\right|=0$. Note that $\Sigma_{a c}$ is determined up to sets of Lebesgue measure zero

Received by the editors June 14, 2010

2010 Mathematics Subject Classification. Primary 42C05, 47B36, 81Q10.

Key words and phrases. Reflectionless Jacobi matrix, Denisov-Rakhmanov Theorem.

The author's work was supported by NSF grant DMS 0758594.

(C)2010 American Mathematical Society Reverts to public domain 28 years from publication 
by this condition. The absolutely continuous spectrum, $\sigma_{a c}$, can be obtained from $\Sigma_{a c}$ by taking the essential closure (of an arbitrary representative).

In general, both assumptions of the DR Theorem are needed. However, in the Schrödinger case $\left(a_{n} \equiv 1\right)$, assumption (2) can be dropped. This surprising result is due to Damanik, Hundertmark, Killip, and Simon [3].

In this paper we show that it is also possible to drop assumption (1) instead, and this in fact works in more general situations, not just for Schrödinger operators. The basic tool will be a simple, but perhaps also somewhat surprising, observation about reflectionless Jacobi matrices. Recall that a whole-line Jacobi matrix $J$ is called reflectionless on a Borel set $M \subset \mathbb{R}$ of positive Lebesgue measure if

$$
\operatorname{Re} g_{n}(t)=0 \quad \text { for Lebesgue almost every } t \in M
$$

and for all $n \in \mathbb{Z}$. Here $g_{n}(z)=\left\langle\delta_{n},(J-z)^{-1} \delta_{n}\right\rangle$ is the Green function of $J$ at site $n$, and $g_{n}(t)=\lim _{y \rightarrow 0+} g_{n}(t+i y)$; this limit exists for almost every $t \in \mathbb{R}$. See [11] for more information on reflectionless operators and why they are important.

We will denote the set of reflectionless (on $M$ ) Jacobi matrices by $\mathcal{R}(M)$.

Theorem 1.2. Suppose that $J \in \mathcal{R}(I)$ with $I=[B-2 A, B+2 A]$. Then $a_{n} \geq A$ for all $n \in \mathbb{Z}$. If $a_{n_{0}}=A$ for a single $n_{0} \in \mathbb{Z}$, then $a_{n}=A, b_{n}=B$ for all $n \in \mathbb{Z}$.

Note that we do not make any assumptions on the spectral behavior of $J$ outside the interval $I$; the spectrum of $J$ could be much bigger than this set, and there is no restriction on the type of this additional spectrum (if present). $\mathcal{R}(I)$ is a large space, and it contains many familiar operators, for example solitons and all periodic operators whose spectrum contains $I$.

Theorem 1.2 implies the promised Schrödinger version of the DR Theorem without hypothesis (1). The proof is very easy if we follow the treatment of the DR Theorem that was given in [11. The idea was to show that the $\omega$ limit set, defined as the collection of limit points $\lim S^{n_{j}} J_{+}$, consists of the free Jacobi matrix $\left(a_{n} \equiv 1, b_{n} \equiv 0\right)$ only. Here $S$ denotes the shift map, and we take limits with respect to pointwise convergence of the coefficients. Again see [11 for more background information.

So suppose now that $a_{n} \equiv 1$ and $\Sigma_{a c}\left(J_{+}\right)=[-2,2]$, and let $J_{0} \in \omega\left(J_{+}\right)$. Then clearly $J_{0}$ has coefficients $a_{n}\left(J_{0}\right) \equiv 1$ also; moreover, $J_{0} \in \mathcal{R}([-2,2])$ by [11, Theorem 1.4]. Hence $b_{n}\left(J_{0}\right) \equiv 0$ by Theorem 1.2 which is what we wanted to show.

The same argument establishes the following stronger result.

Theorem 1.3. Suppose that $\Sigma_{a c}\left(J_{+}\right) \supset[-2,2]$. Furthermore, suppose that there exists a subsequence $n_{j} \rightarrow \infty$ with bounded gaps (that is, $\sup \left(n_{j+1}-n_{j}\right)<\infty$ ) so that $a_{n_{j}} \rightarrow 1$. Then

$$
a_{n} \rightarrow 1, b_{n} \rightarrow 0 \quad(n \rightarrow \infty)
$$

Here is another (related) immediate consequence of Theorem 1.2

Theorem 1.4. Suppose that $\Sigma_{a c}\left(J_{+}\right) \supset[B-2 A, B+2 A]$. Then

$$
\liminf _{n \rightarrow \infty} a_{n} \geq A .
$$

Proof. By [11, Theorem 1.4], every $J_{0} \in \omega\left(J_{+}\right)$satisfies $J_{0} \in \mathcal{R}(I)$. By Theorem 1.2. this implies that $a_{n}\left(J_{0}\right) \geq A$ for all $n \in \mathbb{Z}$. If (1.1) did not hold, then there would be $\omega$ limit points whose coefficients violate this inequality. 
It was previously known that $\lim \inf \left(a_{1} \cdots a_{n}\right)^{1 / n} \geq A$ in the situation of Theorem 1.4, see [4, Theorem 5.7] for a closely related statement and [12] for the use of potential theoretic tools in this context.

Theorem 1.2 also generalizes certain uniqueness results for ergodic operators from 4], but we will not make this explicit. Finally, by making use of basic continuity properties, we can formulate an Oracle Theorem type version of Theorem 1.2 .

Theorem 1.5. Fix $I$ as above and let $R>|B|+2 A$. Then, for every $\epsilon>0$ and $L \in \mathbb{N}$, there exists $\delta>0$ such that if $J \in \mathcal{R}(I),\|J\| \leq R$, and $a_{n_{0}}<A+\delta$ for some $n_{0} \in \mathbb{Z}$, then

$$
A \leq a_{n}<A+\epsilon, \quad\left|b_{n}-B\right|<\epsilon \quad\left(n_{0}-L \leq n \leq n_{0}+L\right)
$$

Notice that this is uniform in the sense that $\delta$ depends on $\epsilon, L, R, A$, but not on $J$ (or $n_{0}, B$ ). The same statement is obtained for all sufficiently large $n_{0}$ if, instead of $J \in \mathcal{R}(I)$, we just assume that $\Sigma_{a c}\left(J_{+}\right) \supset I$; this variant immediately follows from [11, Theorem 1.4] again, which shows that $\omega\left(J_{+}\right) \subset \mathcal{R}(I)$. Here is still another way of saying this: If $\Sigma_{a c}\left(J_{+}\right) \supset I$ and $a_{n_{j}} \rightarrow A$ along some subsequence $n_{j} \rightarrow \infty$, then there are $L_{j} \rightarrow \infty$ so that

$$
\lim _{j \rightarrow \infty} \sup \left\{\left|a_{k}-A\right|+\left|b_{k}-B\right|:\left|k-n_{j}\right| \leq L_{j}\right\}=0
$$

(if $a_{n_{0}}$ is close to $A$, then $\left(a_{n}, b_{n}\right)$ are close to $(A, B)$ on a long interval centered at $\left.n_{0}\right)$. Clearly, Theorem 1.3 is an immediate consequence of this in turn; all these results are closely related.

We present the proofs of this and Theorem 1.2 in the following section. In the final section, we discuss a generalization of Theorem [1.2, where we replace the interval $I$ by a general compact set. This yields a general version of Theorem 1.4 . and this may be interpreted as a quantitative version of the theorem of Dombrowski and of Simon and Spencer [6], [13] on the absence of absolutely continuous spectrum. We discuss all this in Section 3.

\section{Proof of Theorems 1.2 and 1.5}

We will make use of the inverse spectral theory for reflectionless operators in the form presented in [9], 11] (but see also [2], 7], [14, among others, where such tools were used much earlier). We will review some aspects of this theory, but please also consult [9], 11], 14] for further details.

Proof of Theorem 1.2. By rescaling and shifting, it of course suffices to discuss the case $A=1, B=0$. So let $J \in \mathcal{R}([-2,2])$. Consider its $H$ function $H(z)=-1 / g_{0}(z)$ (compare [9], [1]) and the associated Krein function

$$
\xi(t)=\frac{1}{\pi} \lim _{y \rightarrow 0+} \operatorname{Im} \ln H(t+i y) .
$$

Since $J$ is reflectionless on $(-2,2)$, we have that $\xi=1 / 2$ on this set; see, for example, 9, Proposition 2.1]. Fix $R>0$ so large that $\sigma(J) \subset[-R, R]$. Then the exponential Herglotz representation of $H$ reads

$$
H(z)=(z+R) \exp \left(\int_{-R}^{R} \frac{\xi(t) d t}{t-z}\right) .
$$


The $H$ function $H_{0}(z)=\sqrt{z^{2}-4}$ of the free Jacobi matrix $\left(a_{n} \equiv 1, b_{n} \equiv 0\right)$ has a similar representation; here we can take $R=2$, and, of course, $\xi_{0}(t)=\xi(t)=1 / 2$ on $t \in(-2,2)$. Thus

$$
H(z)=H_{0}(z) h(z)
$$

where

$$
h(z)=\exp \left(\int_{-R}^{-2} \frac{\xi(t)-1}{t-z} d t+\int_{2}^{R} \frac{\xi(t) d t}{t-z}\right) .
$$

As a Herglotz function, $H$ has a unique associated (finite, compactly supported) measure $\rho$; for example, we can obtain $\rho$ as the weak-* limit

$$
d \rho(t)=\frac{1}{\pi} \lim _{y \rightarrow 0+} \operatorname{Im} H(t+i y) d t
$$

Since $J \in \mathcal{R}([-2,2])$, we have that $\operatorname{Im} H(t)=|H(t)|$ almost everywhere on $(-2,2)$, and this function is the density of $\pi \rho_{a c}$ on this set. The Jacobi matrices from $\mathcal{R}([-2,2])$ are in one-to-one correspondence with the half-line spectral measures $\nu_{+}$ of the following type:

$$
d \nu_{+}(t)=\frac{1}{2} \chi_{(-2,2)}(t) d \rho_{a c}(t)+f(t) d \rho(t)
$$

where $0 \leq f \leq 1$ and $f=0$ on $(-2,2)$. See [9, Section 2] and/or [11, Section 5]. Rewrite this as

$$
d \nu_{+}(t)=\frac{1}{2 \pi} \chi_{(-2,2)}(t)|H(t)| d t+d \mu(t)
$$

If $J=J_{0}$ is the free Jacobi matrix, then the corresponding measure is given by

$$
d \nu_{+}^{(0)}(t)=\frac{1}{2 \pi} \chi_{(-2,2)}(t)\left|H_{0}(t)\right| d t
$$

so we can also say that

$$
d \nu_{+}(t)=|h(t)| d \nu_{+}^{(0)}(t)+d \mu(t) .
$$

Now by direct inspection of (2.2), we easily confirm that $|h(x)| \geq 1$ for $-2<x<2$; indeed, both integrals from the exponent are non-negative for these $z=x$. Thus

$$
\nu_{+}(\mathbb{R}) \geq \nu_{+}^{(0)}(\mathbb{R})=1
$$

We have the general formula $\nu_{+}(\mathbb{R})=a_{0}^{2}$; compare the discussion of formula $(2.3)$ from 9] or see Sections 2.1 and 2.7 of [14]. This gives the inequality stated in Theorem 1.2 (for $n=0$, but of course we can consider shifts of $J$ to obtain the statement for general $n \in \mathbb{Z}$ ). Moreover, if $a_{0}=1$, then $|h(x)|=1$ almost everywhere on $x \in(-2,2)$, but then another look at (2.2) reveals that $\xi=1$ on $(-R,-2)$, $\xi=0$ on $(2, R)$. So $h \equiv 1$ and thus $H=H_{0}, \nu_{+}=\nu_{+}^{(0)}$, and since $\nu_{+} \operatorname{determines} J$, as mentioned earlier, we conclude that $J$ is the free Jacobi matrix $J_{0}$.

Proof of Theorem 1.5, To obtain Theorem 1.5 from this argument, notice that

$$
\left\|\nu_{+}-\nu_{+}^{(0)}\right\|=\nu_{+}(\mathbb{R})-\nu_{+}^{(0)}(\mathbb{R})=a_{0}^{2}-1 .
$$

This follows because (2.4) shows that $d \nu_{+}^{(0)}=g d \nu_{+}$with $g \leq 1$. The map $\nu_{+} \mapsto J$, restricted to those $\nu_{+}$for which the corresponding $J \in \mathcal{R}(I)$ satisfies $\|J\| \leq R$, is uniformly continuous if we use a metric that induces the weak- $*$ topology on the $\nu_{+}$ 
and pointwise convergence of the coefficients for the Jacobi matrices $J$; explicitly, we may use the metric

$$
d\left(J, J^{\prime}\right)=\sum_{n \in \mathbb{Z}} 2^{-|n|}\left(\left|a_{n}-a_{n}^{\prime}\right|+\left|b_{n}-b_{n}^{\prime}\right|\right)
$$

on the image. See [9, Proposition 2.3] for these statements. Theorem 1.5 follows.

\section{Reflectionless operators on GEnERAL SETS}

We now present a generalization of Theorem 1.2 instead of intervals $I$, we now consider arbitrary compact sets $K$. We will of course insist that $K$ have positive Lebesgue measure; otherwise, the condition of being reflectionless on $K$ becomes vacuous. We must first decide on a proper replacement for the Jacobi matrix with constant coefficients $a_{n}=A, b_{n}=B$. It is natural to proceed as follows. For a compact set $K \subset \mathbb{R}$, define

$$
\mathcal{R}_{0}(K)=\{J \in \mathcal{R}(K): \sigma(J) \subset K\} .
$$

This is a compact set itself if we again use the metric $d$ from (2.5). If $K=I=$ $[B-2 A, B+2 A]$, then $\mathcal{R}_{0}(K)$ consists of a single operator, the Jacobi matrix with constant coefficients.

Please see also [8] and especially [9] for a rather extensive discussion of various aspects of these spaces.

Theorem 3.1. Fix a compact set $K \subset \mathbb{R}$ of positive Lebesgue measure. Then there exists a constant $A=A(K)>0$ such that the following holds: If $J \in \mathcal{R}(K)$, then $a_{n} \geq A$ for all $n \in \mathbb{Z}$. Moreover, if $a_{n_{0}}=A$ for a single $n_{0} \in \mathbb{Z}$, then $J \in \mathcal{R}_{0}(K)$ (and there are such minimizing operators $J_{0} \in \mathcal{R}_{0}(K)$ for which $a_{n_{0}}\left(J_{0}\right)=A$ ).

As we will see, this is easy to prove with the same methods. Note, however, that the minimizing $J_{0}$ 's can easily form a small subset of $\mathcal{R}_{0}(K)$. There are simple examples (periodic operators) where most $J \in \mathcal{R}_{0}(K)$ satisfy the strict inequalities $a_{n}(J)>A$.

It would be interesting to investigate if one could also treat all operators from $\mathcal{R}_{0}(K)$ on an equal footing, at least in certain well-studied cases (finite gap sets $K)$. One could try to find a function $F(a, b)$ of the coefficients that characterizes $\mathcal{R}_{0}(K)$ among all operators from $\mathcal{R}(K)$ in the sense that $F\left(S^{n} a, S^{n} b\right) \geq F_{0}$ for all $J \in \mathcal{R}(K)$ and all $n \in \mathbb{Z}$ ( $S$ denotes the shift), and we have equality for a single $n_{0} \in \mathbb{Z}$ precisely if $J \in \mathcal{R}_{0}(K)$. This can reasonably be expected to hold only if $K$ is an essentially closed set (consider solitons!).

Theorem 3.1 does include Theorem 1.2 as a special case, because, as discussed, if $K=[B-2 A, B+2 A]$, then only the Jacobi matrix with constant coefficients $a_{n}=A, b_{n}=B$ lies in $\mathcal{R}_{0}(K)$.

Proof. We follow the strategy from Section 2, suitably adjusted. We can simply define

$$
A=\inf _{J \in \mathcal{R}_{0}(K)} a_{0}(J) .
$$

Since $\mathcal{R}_{0}(K)$ is a compact metric space and $J \mapsto a_{0}(J)$ is a continuous map, $A$ is actually a minimum. In particular, $A>0$ (one can of course consider Jacobi matrices with zero $a$ 's, but it is easy to see that such an operator cannot be reflectionless on a positive measure set). 
We must now show that if $J \in \mathcal{R}(K)$, then $a_{0}(J) \geq A$, and we will have strict inequality here unless $J \in \mathcal{R}_{0}(K)$. Since both $\mathcal{R}(K)$ and $\mathcal{R}_{0}(K)$ are shift invariant, this will yield the theorem.

The $\xi$ function of a $J \in \mathcal{R}(K)$ satisfies $\xi=1 / 2$ on $K$, and of course we have that $0 \leq \xi \leq 1$. Fix a bounded component $I=(c, d)$ of the open set $K^{c}$. We now claim that if we replace $\xi$ with

$$
\xi_{1}(t)= \begin{cases}\chi_{(d-g, d)}(t), & t \in I, \\ \xi(t), & t \notin I,\end{cases}
$$

where $g=\int_{c}^{d} \xi d t$, then $\left|H_{1}(x)\right| \leq|H(x)|$ for almost every $x \notin(c, d)$. More precisely, recall that we define $H(x)=\lim _{y \rightarrow 0+} H(x+i y)$ for $x \in \mathbb{R}$, and this limit exists almost everywhere; we obtain the inequality for all $x \notin[c, d]$ for which $H(x), H_{1}(x)$ can be defined in this way. The proof of this claim is easy; use the representation (2.1) to compare $H$ and $H_{1}$. The statement is also formulated as [8, Lemma 2.5], and a detailed formal proof can be found there.

We now want to modify $\xi$ in this way on all bounded components of $K^{c}$, and we also want to put $\xi=1$ to the left of $K$ and $\xi=0$ to the right of $K$. The plan is to pass from $\xi$ to a new Krein function $\xi_{0}$ in this way through a series of intermediate functions $\xi_{n}$, and we expect that $\left|H_{0}(x)\right| \leq|H(x)|$ for almost every $x \in K$, because $\left|H_{n}(x)\right|$ goes down at each individual step.

To make this rigorous, it is best to work with the Hilbert transform

$$
(T \xi)(x)=\lim _{y \rightarrow 0+} \int_{|t-x|>y ;|t|<R} \frac{\xi(t) d t}{t-x} ;
$$

here $R>0$ is again chosen so large that $\sigma(J) \subset[-R, R]$. We use the following basic facts; please consult [1] for background information. The limit defining the Hilbert transform exists for almost every $x \in \mathbb{R}$, and

$$
|H(x)|=(x+R) e^{(T \xi)(x)}
$$

almost everywhere. Moreover, if $\xi_{n} \rightarrow \xi$ in $L^{2}(-R, R)$, then also $T \xi_{n} \rightarrow T \xi$ in $L^{2}(\mathbb{R})$.

Now put $\widetilde{\xi}=1$ to the left of $K, \widetilde{\xi}=0$ to the right of $K$, and $\widetilde{\xi}=\xi$ otherwise. It is obvious that $T \widetilde{\xi} \leq T \xi$ (almost everywhere) on $K$. Let $\left\{I_{n}\right\}_{n \geq 1}$ be a complete list of the bounded components of $K^{c}$; if there are only finitely many components, then no limiting process is necessary and this part of the discussion becomes much easier. Modify $\widetilde{\xi}$ on $I_{1}$ in the way described above to obtain $\xi_{1}$. As we saw earlier, we then have that $T \xi_{1} \leq T \widetilde{\xi}$ almost everywhere on $K$. Continue in this way; construct $\xi_{n+1}$ from $\xi_{n}$ by modifying this function on $I_{n+1}$. Clearly, $\xi_{n}$ converges in $L^{1}(-R, R)$ (and thus also in $L^{2}$, since $0 \leq \xi_{n} \leq 1$ ) to a limiting function $\xi_{0}$. The Hilbert transforms converge in $L^{2}(-R, R)$ and also pointwise almost everywhere on $K:\left(T \xi_{n}\right)(x) \rightarrow\left(T \xi_{0}\right)(x)$ (it is not necessary to pass to a subsequence because this sequence is decreasing almost everywhere on $K)$. Hence $\left(T \xi_{0}\right)(x) \leq(T \xi)(x)$ for almost every $x \in K$. The Krein function $\xi_{0}$ is a step function on each gap $I_{n}$, and it jumps from 0 to 1 (or not at all). Moreover, $\xi_{0}=1 / 2$ on $K$, and $\xi_{0}=1$ to the left of $K$ and $\xi_{0}=0$ to the right of $K$. In [9], we introduced the notation $X(K)$ for the set of all Krein functions of this type, so we can summarize by writing $\xi_{0} \in X(K)$. The significance of $X(K)$ comes from the fact that $X(K)$ is exactly the collection 
of Krein functions of operators from $\mathcal{R}_{0}(K)$; see [9, Section 2] for this statement, especially the discussion of equation (2.6) of $[9$.

Recall that for any $J \in \mathcal{R}(K)$, we have that

$$
\pi \rho_{a c}(K)=\int_{K}|H(x)| d x
$$

where $\rho$ refers to the measure associated with the $H$ function of $J$, as in (2.3). Thus, in terms of $\rho$, what we have just shown says that if $J \in \mathcal{R}(K)$, then there exists $\xi_{0} \in X(K)$ so that

$$
\rho_{a c}(K) \geq \rho_{a c}^{(0)}(K),
$$

where $\rho^{(0)}$ is the measure associated with (the $H$ function of) $\xi_{0}$. Now recall how the half-line spectral measures $\nu_{+}$were obtained from $\rho$ : We have that $\nu_{+}=f \rho$, where $f=1 / 2$ Lebesgue almost everywhere on $K$ and $0 \leq f \leq 1$, and every such $f$ is admissible. Thus $\nu_{+}(\mathbb{R}) \geq(1 / 2) \rho_{a c}(K)$. Moreover, if $\xi_{0} \in X(K)$ and we take $f=0$ off the support of $\rho_{a c}^{(0)}$, then the corresponding $\nu_{+}^{(0)}=(1 / 2) \chi_{K} \rho_{a c}^{(0)}$ belongs to a Jacobi matrix $J_{0} \in \mathcal{R}_{0}(K)$. See again [9, Section 2] for this description of the spectral data of $\mathcal{R}_{0}(K)$.

The upshot of all this is that (3.1) implies that for arbitrary $J \in \mathcal{R}(K)$, there exists $J_{0} \in \mathcal{R}_{0}(K)$ so that

$$
\nu_{+}(\mathbb{R}) \geq \nu_{+}^{(0)}(\mathbb{R}) .
$$

In other words, $a_{0}(J) \geq a_{0}\left(J_{0}\right) \geq A$, as we wanted to show.

It remains to discuss the case of equality. Clearly, this can only happen if $T \xi_{0}=$ $T \xi$ almost everywhere on $K$ in the construction above, but at each individual step, when going from $\xi_{n}$ to $\xi_{n+1}$, the Hilbert transform will decrease stricly almost everywhere on $K$ unless $\xi_{n}=\xi_{n+1}$. Therefore, $T \xi_{0}=T \xi$ on $K$ implies that $\xi=\xi_{0}$, and thus $\xi \in X(K)$. Moreover, even for fixed $\rho$, the half-line spectral measure $\nu_{+}(\mathbb{R})=a_{0}^{2}$ is of course minimized only by $\nu_{+}=(1 / 2) \chi_{K} \rho_{a c}$. As just explained, this implies that $J \in \mathcal{R}_{0}(K)$.

Finally, here is the promised quantitative version of the result of Dombrowski and of Simon and Spencer [6], [13]. These authors show by a decoupling argument that $\liminf a_{n}>0$ if $\sigma_{a c}\left(J_{+}\right) \neq \emptyset$.

Theorem 3.2. Let $M \subset \mathbb{R}$ be a bounded Borel set of positive Lebesgue measure. Then there exists a constant $A=A(M)>0$ such that

$$
\liminf _{n \rightarrow \infty} a_{n} \geq A
$$

for every half-line Jacobi matrix $J_{+}$with $\Sigma_{a c}\left(J_{+}\right) \supset M$.

Proof. Fix a compact subset $K \subset M$ of positive Lebesgue measure, and let $A=$ $A(K)>0$ be the constant from Theorem 3.1. Then $a_{n}(J) \geq A$ for all (whole-line operators) $J \in \mathcal{R}(M) \subset \mathcal{R}(K)$. Since $\omega\left(J_{+}\right) \subset \mathcal{R}(M)$ by [11, Theorem 1.4], we obtain (3.2) from this.

\section{REFERENCES}

[1] J.A. Cima, A.L. Matheson, and W.T. Ross, The Cauchy Transform, Mathematical Surveys and Monographs, 125, American Mathematical Society, Providence, RI, 2006. MR 2215991 (2006m:30003)

[2] W. Craig, The trace formula for Schrödinger operators on the line, Comm. Math. Phys. 126 (1989), 379-407. MR 1027503 (90m:47063) 
[3] D. Damanik, D. Hundertmark, R. Killip, and B. Simon, Variational estimates for discrete Schrödinger operators with potentials of indefinite sign, Comm. Math. Phys. 238 (2003), 545-562. MR1993385 (2004i:81068)

[4] P. Deift and B. Simon, Almost periodic Schrödinger operators. III. The absolutely continuous spectrum in one dimension, Comm. Math. Phys. 90 (1983), 389-411. MR719297 (85i:34009b)

[5] S. Denisov, On Rakhmanov's theorem for Jacobi matrices, Proc. Amer. Math. Soc. 132 (2004), 847-852. MR2019964(2005h:47060)

[6] J. Dombrowski, Quasitriangular matrices, Proc. Amer. Math. Soc. 69 (1978), 95-96. MR 0467373 (57:7232)

[7] S. Kotani, Generalized Floquet theory for stationary Schrödinger operators in one dimension, Chaos Sol. Fract. 8 (1997), 1817-1854. MR1477262 (98m:34173)

[8] A. Poltoratski and C. Remling, Reflectionless Herglotz functions and Jacobi matrices, Comm. Math. Phys. 288 (2009), 1007-1021. MR.2504863 (2010i:47063)

[9] A. Poltoratski and C. Remling, Approximation results for reflectionless Jacobi matrices, to appear in Int. Math. Res. Notices, http://arxiv.org/abs/1005.2149.

[10] E.A. Rakhmanov, The asymptotic behavior of the ratio of orthogonal polynomials. II (Russian), Mat. Sb. (N.S.) 118(160) (1982), 104-117. MR654647 (83m:42018)

[11] C. Remling, The absolutely continuous spectrum of Jacobi matrices, to appear in Annals of Math.

[12] B. Simon, Equilibrium measures and capacities in spectral theory, Inverse Probl. Imaging 1 (2007), 713-772. MR2350223 (2008k:31003)

[13] B. Simon and T. Spencer, Trace class perturbations and the absence of absolutely continuous spectra, Comm. Math. Phys. 125 (1989), 113-125. MR.1017742 (91g:81018)

[14] G. Teschl, Jacobi Operators and Completely Integrable Nonlinear Lattices, Mathematical Monographs and Surveys, 72, American Mathematical Society, Providence, RI, 2000. MR 1711536 (2001b:39019)

Department of Mathematics, University of Oklahoma, Norman, Oklahoma 73019

E-mail address: cremling@math.ou.edu

$U R L$ : www. math.ou.edu/ cremling 